\title{
Resection of a Meningioma at Craniocervical Junction through Far Lateral Approach: Two- Dimensional Operative Video
}

\author{
Yong Yan ${ }^{1, *}$ Hongxiang Wang ${ }^{1, *}$ Tao Xu ${ }^{1}$ Zhenyu Gong ${ }^{1} \quad$ Fan Hong $^{1} \quad \mathrm{Xu}_{\text {Zhang }}{ }^{1}$ Juxiang Chen ${ }^{1}$ \\ ${ }^{1}$ Department of Neurosurgery, Changzheng Hospital, Naval Medical \\ University, Neurosurgical Institute of Shanghai, Neurosurgical \\ Address for correspondence Juxiang Chen, MD, PhD, Department of \\ Institute of PLA, Shanghai, China \\ Neurosurgery, Changzheng Hospital, Naval Medical University, 415 \\ Fengyang Road, Shanghai, China (e-mail: juxiangchen@smmu.edu.cn).
}

J Neurol Surg B 2019;80(suppl S4):S358-S359.

\begin{abstract}
Tumors located in the craniocervical junction region are significantly challenging for surgical resection. We shared our experience of a meningioma at craniocervical junction resected through far lateral approach in a 68-year-old female. The patient presented with intermittent headache with discomfort in the neck and shoulders for 3 years without any positive signs. Magnetic resonance imaging (MRI) revealed a tumor of $3.6 \mathrm{~cm} \times 3.0 \mathrm{~cm} \times 2.5 \mathrm{~cm}$ lying at the ventral side of medulla oblongata, with T1 hypointensity, $\mathrm{T} 2$ hyperintensity, and a significant enhancement on T1-contrast image. The far lateral approach on the right side was planned to resect the tumor with a parkbench position. The patient underwent a standard craniotomy using a lazy S-shaped incision. The transposition of vertebral artery was performed carefully therein, followed by removal of part of the arches of atlas and axis. After exposure of the tumor, vertebral artery (VA) and posterior inferior cerebellar artery (PICA) adhesive to the lesion could be seen operatively. Truncating the supplying blood vessels of the tumor was taken as the first step, followed by resecting the tumor mass in a piecemeal manner. While preserving VA, PICA, posterior nerves, medulla oblongata, and cervical cord, gross-total resection was achieved under the careful operation. The patient

\section{Keywords}

- operative video

- meningioma

- craniocervical junction

- far lateral approach tolerated the procedure well without any neurological deficits. Histological examination confirmed the tumor as a meningioma (World Health Organization [WHO] grade I). Postoperative MRI scan depicted complete resection of the tumor. The patient remained symptom free without any evidence of recurrence during the follow-up period of 1 year. Informed consent was obtained from the patient. The link to the video can be found at: https://youtu.be/i9H-wS4fF10.
\end{abstract}

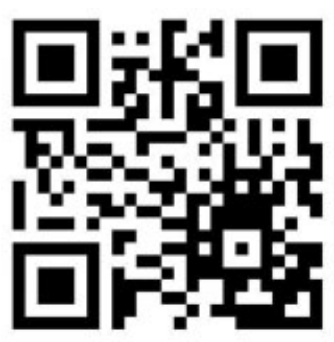

These authors contributed equally to this work.

received

April 22, 2019

accepted after revision

August 24, 2019

published online

October 23, 2019 www.thieme.com/skullbasevideos

www.thieme.com/jnlsbvideos

(c) 2019 Georg Thieme Verlag KG Stuttgart · New York
License terms

c) $(1) \$$ 


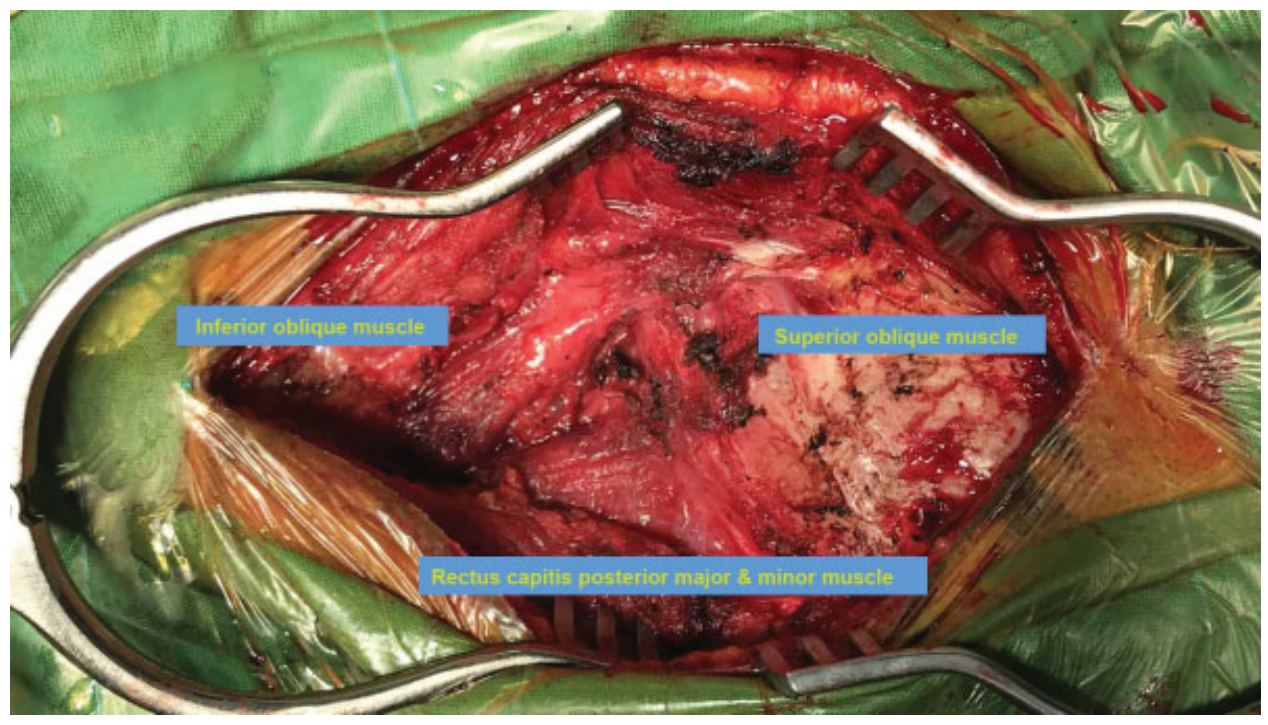

Fig. 1 Exposure of the suboccipital triangle to distinguish vertebral artery.

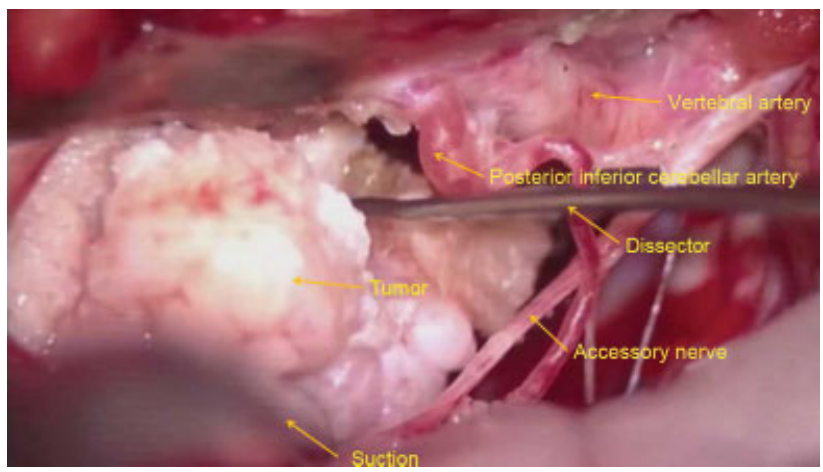

Fig. 2 Intraoperative image shows the exact position and adjacent structures of the tumor.

\section{Conflicts of Interest}

None declared. 